\title{
Topology, Unitary Representations and Charged Particles
}

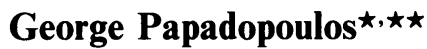

Mathematics Department, King's College London, London WC2R 2LS, UK

Received August 10, 1990; in revised form August 5, 1991

\begin{abstract}
The quantum mechanics of the charged particles with rigid and local symmetries propagating on the manifold $M$ is studied. It is shown that the classical rigid symmetries of this model may be anomalous. These anomalies are of local and global type, and they related to topological obstructions to lifting a group action of a group $G$ on $M$ to a principal $U(1)$ bundle $P$ over $M$. The charged particles with local symmetries may have additional anomalies and the representation theory of the group $G$ is used to study these anomalies. Finally, the quantum mechanics of the supersymmetric charged particles with symmetries is examined.
\end{abstract}

\section{Introduction}

The classical and quantum theory of a charged particle coupled to a $U(1)$ gauge field (magnetic field) has attracted much attention. This is due to the existence of quantum mechanical properties of this model which have topological interpretation.

There are two such quantum properties. The first one is Dirac's observation that the coupling constant of gauge field must be quantised [1]. The second property is the observation of the authors in refs. [2-4] that the symmetries of the classical equations of motion of this theory might be anomalous. In ref. [2], N. S. Manton observed that a $U(1)$ symmetry of a charged particle propagating on a flat torus is anomalous. In ref. [3], R. S. Ward extended this result to the $U(1)$ symmetries of a charged particle propagating on any manifold $M$. The anomaly was contributed to the fact that the $U(1)$ charges at the quantum level are locally defined operators. These anomalies were also observed in ref. [10] using path integral methods. Finally in ref. [4], R. Jackiw suggested that the algebra of

\footnotetext{
* e. mail address: udah130@oak.cc.kcl .ac.uk

$\star \star$ Address after September 1990: Physics Department, Queen Mary and Westfield College, Mile End, London E1 4NS
} 
charges associated with the symmetries of a charged particle may develope a central extension even that the classical level.

In ref. [5], it was observed that these anomalies have a topological interpretation. They are related to obstructions to lifting a group action $f_{g}$ of a group $G$ on $M$ to a $U(1)$ principal bundle $P=P(M, U(1), \pi)$ over $M$ with projection $\pi$ and by using topological methods a refinement of the results of refs. [2-4] was obtained The group action $f_{g}$ generates the classical rigid symmetries of the model. $P$ arises naturally in the quantum theory of the charged particle from Dirac's quantisation condition.

In this paper, the aim is to emphasise the importance of global and topological properties of the manifold $M$ in which the charged particle propagates in the quantisation of this theory and to provide a unified treatment of the quantum properties of the (supersymmetric) charged particle with symmetries. The main result of this paper is the proof of the correspondence between the anomalies in the rigid symmetries of a charged particle and the topological obstructions to lifting a group action $f_{g}$ to a principal bundle $\mathrm{P}$. It is shown that there are both local and global type of anomalies. Several examples of anomalies are given.

A subgroup $G^{\prime}$ of the rigid symmetry group $G$ of a charged particle is gauged. Then it is shown that the gauged charged particle may have additional anomalies apart from those of the charged particles with rigid symmetries. These anomalies exist whenever the Hilbert space of the corresponding quantum mechanical model with the rigid symmetries does not carry the trivial representation of $G^{\prime}$ as subgroup of $G$. If this anomaly is present, the imposition of the physical states condition for a gauged charged particle leads to an empty physical Hilbert space. Finally, the quantum mechanics of the $N=1$ supersymmetric charged particle propagating on a manifold $M$ with a group action $f_{g}$ is studied. In this case, the anomalies in the symmetries of the.model generated by $f_{g}$ are related to the obstructions to lifting the group action to a principal bundle $P=P(M, K, \pi)$ over $M$ with fibre a (non-abelian) group $K$. Moreover, it is shown that some of the anomalies of the supersymmetric charged particle correspond to obstructions of a topological extension problem.

In Sect. 2, the classical theory of the charged particle with rigid and local symmetries is considered. Particular attention is paid on the global and topological issues that arise at the classical level.

In Sect. 3, the quantisation of the theory with rigid and local symmetries is studied. It is shown how the liftings of the group action $f_{g}$ of the group $G$ can be used to construct unitary representations of $G$ on the Hilbert space of the theory and the correspondence between anomalies and topological obstructions is established.

In Sect. 4, the anomalies of the models with rigid symmetries are examined. This is achieved by using the results of the appendices regarding the obstructions to lifting group actions on principal bundles. Several examples of local and global anomalies are given. In Sect. 5 , the supersymmetric charged particle with rigid symmetries is considered. For this model it is shown that some of its anomalies correspond to obstructions of a topological extension problem.

In Sect. 6, the spectrum of the Hamiltonian operator of the charged particles propagating on any homogeneous space $G / H$ is presented where $G$ is any compact, connected and semisimple Lie group. The standard left action of $G$ on $G / H$ is gauged and an example of a gauged model with empty physical Hilbert space is 
given. In Sect. 7, we give our conclusions and include a comment on the imposition of classical symmetries to the quantum sectors associated to different $\theta$-structures [12] that appear in the quantisation of the charged particle.

In Appendix A1, the problem of lifting a group action $f_{g}$ of a compact Lie group $G$ on $M$ to a principal bundle $P=P(M, K, \pi)$ over $M$ is stated and the necessary conditions for the existence of a lifting are given. In Appendix A2, the Hattori-Yoshida theorem $[6,7]$ that describes the necessary and sufficient conditions to lifting a group action $f_{g}$ of a compact Lie group $G$ on a manifold $M$ to a principal $U(1)$ bundle $P=P(M, U(1), \pi)$ over $M$ is presented. The obstructions are studied using (continuous) group cohomology [8] and singular cohomology. In Appendix A3, a simple way to describe the obstructions to lifting the group action of any compact and connected group $G$ to a principal $U(1)$ bundle $P(M, U(1), \pi)$ is given. Finally in Appendix A4, the lifting of the left group action of $G$ on the homogeneous space $G / H$ to any principal bundle $P=P(M, K, \pi)$ over $G / H$ is briefly examined [9]. The lifting problem of group actions to principal bundles with discrete fibres is also stated $[17,12]$.

\section{Classical Theory}

The equations of motion of a charged particle propagating on a manifold $M$ are

$$
-\nabla_{t} \partial_{t} \phi^{i}+\mathbf{g}^{i j} \omega_{j k} \partial_{t} \phi^{k}=0, \quad \partial_{t}=\partial / \partial t
$$

where $\phi$ is a $C^{\infty}$ map from a wordline $I=[0,1](t \in I)$ to a $C^{\infty}$ manifold $M$ with metric $\mathbf{g}, i, j=1, \ldots, \operatorname{dim} M . \nabla_{t}=\partial_{t} \phi^{i} \nabla_{i}$ and $\nabla_{i}$ is the Levi-Civita connection of the metric $\mathbf{g}$ of $M$ and $\omega$ is a closed $(d \omega=0)$ but not necessarily exact two form of $M$. The manifold $M$ is restricted to be compact, path connected, oriented and without boundary.

Let $\left\{U_{\alpha}\right\}$ be a good cover of $M$, the Lagrangian of the theory is given by

$$
L_{\alpha}=(1 / 2) \mathbf{g}_{i j \alpha} \partial_{t} \phi_{\alpha}^{i} \partial_{t} \phi_{\alpha}^{j}+b_{i \alpha} \partial_{t} \phi_{\alpha}^{i}
$$

on each $U_{\alpha}$ of $M$ where $\omega_{\alpha}=d b_{\alpha}$ and

$$
b_{\beta}=b_{\alpha}+d w_{\beta \alpha} \quad\left(w_{\alpha \beta}=-w_{\beta \alpha}\right)
$$

are the patching conditions of $\left\{b_{\alpha}\right\} . w_{\alpha \beta}$ is a function defined on the intersection $U_{\alpha \beta}=U_{\alpha} \cap U_{\beta}$ of any two open sets $U_{\alpha}, U_{\beta}$ subsets of $M$. The momentum $p_{i \alpha}=\partial L_{\alpha} / \partial \partial_{t} \phi^{i}$ of the theory is locally defined and obeys the patching conditions

$$
p_{\beta}=p_{\alpha}+d w_{\beta \alpha} \text {. }
$$

The Hamiltonian

$$
H_{\alpha}=(1 / 2) \mathbf{g}_{\alpha}^{i j}\left(p_{i \alpha}-b_{i \alpha}\right)\left(p_{j \alpha}-b_{j \alpha}\right)
$$

is globally defined $\left(H_{\alpha}=H_{\beta}\right)$ under the patching conditions (2.3) and (2.4). Finally the symplectic form $\Omega_{\alpha}=d p_{i \alpha} \wedge d x_{\alpha}^{i}$ is a globally defined two form as well.

To continue, let $f_{g}$ be a group action of a group $G$ on a manifold $M$, i.e. $f_{g} f_{g^{\prime}}=f_{g g^{\prime}}, \forall g, g^{\prime} \in G$ and $f_{e}=\operatorname{Id}_{M} ; e$ is the identity element of the group $G$. The equations of motion (2.1) of the charged particle are invariant under $f_{g}$ provided 
that

$$
f_{g}^{*} \mathbf{g}=\mathbf{g}
$$

and

$$
f_{g}^{*} \omega=\omega, \quad \forall g \in G
$$

where $f_{g}^{*} \mathbf{g}\left(f_{g}^{*} \omega\right)$ is the pullback metric (two-form). Equation (2.6) implies that $f_{g}$, $\forall g \in G$ are isometries, and Eq. (2.7) indicates that the closed two form $\omega$ is invariant under the group action $f_{g}$.

For every group action $f_{g}$ of a compact Lie group $G$, we can always find a metric $\mathbf{g}_{I}$ (two form $\omega_{I}$ ) that satisfies $(2.6)((2.7))$. Indeed given any metric $g$, we set,

$$
\mathbf{g}_{I}=\int_{G} f_{g}^{*} \mathbf{g} d \mu_{H}(g)
$$

where $d \mu_{H}$ is the normalized Haar measure in $G$. Similarly, we can construct $\omega_{I}$. However, if the group $G$ is disconnected the cohomology class $\left[\omega_{I}\right]$ of $\omega_{I}$ in $H^{2}(M, \mathbf{R})$ may not be the same as the class $[\omega]$ of the closed form $\omega$.

Let $k_{a}, a=1, \ldots, \operatorname{dim} G$, be the vector fields on $M$ generated by the group action $f_{g}$. Infinitesimally, Eqs. (2.6) and (2.7) become

$$
L_{a} \mathbf{g}=0
$$

and

$$
L_{a} \omega=0,
$$

where $L_{a}$ is the Lie derivative with respect to the vector field $k_{a} . L_{a}=l_{a} d+d l_{a}$, where $l_{a}$ is the inner product with respect to vector field $k_{a}$ that maps $q$ forms to $q-1$ forms. The conserved charges associated with the symmetries generated by $f_{g}$ are

where

$$
Q_{a \alpha}=k_{a \alpha}^{i}\left(p_{i \alpha}-b_{i \alpha}\right)-m_{a \alpha}
$$

$$
l_{a} \omega_{\alpha}=d m_{a \alpha}
$$

and $m_{a \alpha}$ is a locally defined function on $M$. The charge $Q_{a}$ is globally defined on $M$ provided that $m_{a \alpha}$ is globally defined as well, i.e. $m_{a \alpha}=m_{a \beta}$. The poisson bracket algebra of charges is

$$
\left\{Q_{a \alpha}, Q_{b \alpha}\right\}=-f_{a b}^{c} Q_{c \alpha}+c_{a b \alpha},
$$

where $f_{b c}^{a}$ are the structure constants of the Lie algebra (Lie $G$ ) of the group $G$ and $c_{a b \alpha}$ is a central extension given by

$$
c_{a b \alpha}=l_{a} l_{b} \omega_{\alpha}-f_{a b}^{c} m_{c \alpha} .
$$

$c_{a b}$ is a constant.

To study $m_{a \alpha}$ and the central extension $c_{a b \alpha}$, we define,

$$
\pi_{a r}=\int_{C r} l_{a} \omega(x)
$$

and

$$
\tau_{a b}=\int_{M} l_{a} l_{b} \omega(x) d \mu(x)
$$


where $C_{r}$ are 1-cycles of $M$ and $d \mu$ is the measure in $M$ constructed from the metric $\mathbf{g}$. Both $\pi_{a r}$ and $\tau_{a b}$ are Lie algebra cohomology cocycles [22], i.e. the class of $\pi$ is in $H^{1}\left(\mathrm{Lie} G, H^{1}(M, \mathbf{R})\right)\left(\mathbf{R}\right.$ real numbers) and the class of $\tau$ is in $H^{2}(\operatorname{Lie} G)$. Lie $G$ acts trivially on $H^{1}(M, \mathbf{R})$. If $\pi_{a r}$ vanishes $\left(\pi_{a r}=0\right)$, it implies that $m_{a}$ is globally defined, i.e. $m_{a \alpha}=m_{a \beta}$. Consequently the charge $Q_{a}$ is globally defined as well. Then observe that $c_{a b}$ is cohomologous to $\tau_{a b}$, and if $\tau_{a b}$ represents the trivial class in $H^{2}($ Lie $G)$ after a suitable redefinition of the charges the central extension $c_{a b}$ of the algebra (2.13) vanishes. This concludes the study of the rigid symmetries of the charged particles.

To gauge a subgroup $G^{\prime}$ of the symmetry group $G$, we introduce a gauge field $A$. The Lagrangian of the theory [14] is locally given by

$$
L_{\alpha}=(1 / 2) \mathbf{g}_{i j \alpha} \nabla_{t} \phi_{\alpha}^{i} \nabla_{t} \phi_{\alpha}^{j}+b_{i \alpha} \partial_{t} \phi_{\alpha}^{i}-m_{a^{\prime} \alpha} A^{a^{\prime}},
$$

where

$$
\nabla_{t} \phi_{\alpha}^{i}=\partial_{t} \phi_{\alpha}^{i}+A^{a^{\prime}} k_{a^{\prime} \alpha}^{i}
$$

and $a^{\prime}=1, \ldots, \operatorname{dim} G^{\prime}$. The fields $\phi$ are $C^{\infty}$ sections of the fibre bundle $P\left(I, G^{\prime}, \pi\right) x_{G^{\prime}} M$ over the world line $I=[0,1]$, where $P\left(I, G^{\prime}, \pi\right)$ is a principal bundle over $I$ and $A$ is a connection of $P\left(I, G^{\prime}, \pi\right)$. The Lagrangian (2.17) is gauge invariant (up to surface terms), if the central extension $c_{a^{\prime} b^{\prime} \alpha}(2.13)$ of the algebra of charges is the trivial class in $H^{2}(\operatorname{Lie} G)$. The equations of motion are globally defined provided that $\pi_{a^{\prime} r}(2.15)$ vanishes. The Hamiltonian of the theory is

$$
H_{\alpha}=(1 / 2) \mathbf{g}_{\alpha}^{i j}\left(p_{i}-b_{i}\right)_{\alpha}\left(p_{j}-b_{j}\right)_{\alpha}-A^{a^{\prime}} Q_{a^{\prime} \alpha}
$$

where $Q_{a^{\prime}}, a^{\prime}=1, \ldots, \operatorname{dim} G^{\prime}$, are the charges given by Eq. (2.11). The connection $A$ in (2.19) is a Lagrange multiplier and $Q_{a^{\prime}}$ is a first class constraint that obeys the algebra (2.13) under Poisson brackets. If $G^{\prime}=\left(G^{\prime \prime} \times U(1)\right) / \Gamma, G^{\prime \prime}$ is a subgroup of $G^{\prime}$ and $\Gamma$ a finite group, the Lagrangian (2.17) can be modified by adding a ChernSimons term associated with the $U(1)$ subgroup of $G^{\prime}$.

\section{Quantisation}

To quantise the charged particle propagating on a compact, path connected, oriented manifold $M$ without boundary, we use canonical commutation relations on each patch of the good cover $\left\{U_{\alpha}\right\}$ of $M$ to construct (after a choice of an ordering ambiguity) the locally defined Hamiltonian operator

$$
\hat{H}_{\alpha}=-(1 / 2) \mathbf{g}_{\alpha}^{i j}\left(\nabla_{i \alpha}-i b_{i \alpha}\right)\left(\nabla_{j \alpha}-i b_{j \alpha}\right),
$$

where $\nabla_{i}$ is the Levi-Civita connection of the metric $\mathbf{g}$. The locally defined operators $\left\{\hat{H}_{\alpha}\right\}$ can be patched together $\left(\hat{H}_{\beta}=h_{\beta \alpha} \hat{H}_{\alpha} h_{\alpha \beta}\right)$ to give a globally defined operator $\hat{H}$ on $M$ provided that the following patching conditions hold:

$$
h_{\alpha \beta}=\exp i w_{\alpha \beta}
$$

and

$$
h_{\alpha \beta} h_{\beta \gamma} h_{\gamma \alpha}=1, \quad h_{\beta \alpha}=\left(h_{\alpha \beta}\right)^{-1}
$$

on the overlaps $U_{\alpha \beta}$ and $U_{\alpha \beta \gamma}$ correspondingly. 
The operator $\hat{H}$ acts on the set of $C^{\infty}$ functions $\left\{\psi_{\alpha}\right\}$ which obey the patching conditions

$$
\psi_{\beta}=h_{\beta \alpha} \psi_{\alpha} .
$$

The Hilbert space of wave functions of the theory is the space square integrable sections $L^{2}(\Gamma(L), d \mu)$ of the complex line bundle $L$ with transition functions $\left\{h_{\alpha \beta}\right\}$. $\left\{b_{\alpha}\right\}$ is a connection of $L$. This is Dirac's quantisation condition. The inner product in $L^{2}(\Gamma(L), d \mu)$ is given by

$$
\left(\psi_{1}, \psi_{2}\right)=\int_{M}\left\langle\psi_{1}, \psi_{2}\right\rangle(x) d \mu(x)
$$

where $\langle$,$\rangle is an inner product on the fibers of L$ compatible with the connection $b, d \mu$ is the measure in $M$ which is constructed from the metric $\mathbf{g}$ and $\psi_{1}, \psi_{2} \in L^{2}(\Gamma(L), d \mu) .{ }^{1}$ The operator $\hat{H}$ has a self adjoint extension in the Hilbert space $L^{2}(\Gamma(L), d \mu)$.

Next suppose that the manifold $M$ accepts a group action $f_{g}$ of the group $G$. The Hamiltonian operator $\hat{H}$ remains invariant under $f_{g}$ provided that $f_{g}$ are isometries, $\forall g \in G$, and leave the connection $\left\{b_{\alpha}\right\}$ invariant up to a $U(1)$ gauge transformation. The latter is true, if and only if, there is a lifting $f_{g}^{\uparrow}$ of the group action $f_{g}$ to the principal $U(1)$ bundle $P=P(M, U(1), \pi)$ associated with the line bundle $L$ [15]. Given a lifting $f_{g}^{\uparrow}$ of $G$ on $P$, it is always possible to construct a lifting $f_{g, u}^{\uparrow}$ of $G$ to any associated line bundle $L_{u}=P \times{ }_{u} \mathbf{C}$ of $P$, where $u$ is any unitary irreducible representation of $U(1)$ and $\mathbf{C}$ is the set of complex numbers. The representation $u$ is taken to be irreducible because otherwise $L_{u}$ decomposes to a direct sum of complex line bundles. The lifting $f_{g, u}^{\uparrow}$ is defined by $f_{g, u}^{\uparrow}[p, c]=\left[f_{g}^{\uparrow}(p), c\right]$, where $p \in P$ and $c \in \mathbf{C}$. Using the lifting $f_{g, u}^{\uparrow}$, we can construct a unitary representation $D[12,24]$ of the group $G$ on $L^{2}(\Gamma(L), d v)$ by setting

$$
(D(g) \psi)(x)=\left(\rho\left(g^{-1}, x\right)\right)^{1 / 2} f_{g, u}^{\uparrow} \psi\left(f_{g}^{-1}(x)\right),
$$

where $\psi \in L^{2}\left(\Gamma\left(L_{u}\right), d v\right), d v$ is a quasi-invariant measure on $M$ and $\rho(g, x)$ given by

$$
\rho(g, x)=d v\left(f_{g}(x)\right) / d v(x), \quad \rho>0
$$

is the Radon-Nikodym derivative. In the case of the charged particle with symmetries, $d v$ is set equal to the invariant measure $d \mu$ of the metric $\mathbf{g}$ (Eq. (2.6)) and $\rho=1$.

In conclusion, the symmetries of the charged particle generated by the group action $f_{g}$ of the group $G$ are represented by unitary transformations on the Hilbert space $L^{2}(\Gamma(L), d \mu)$ of the theory, if and only if there is a lifting $f_{g}^{\uparrow}$ of $f_{g}$ on the complex line bundle $L$ that arises from Dirac's quantisation condition. Therefore the obstructions to lifting the group action $f_{g}$ on $M$ to $L$ correspond to the anomalies in the quantisation of this model. These anomalies are examined in Sect. 4.

Having studied the quantisation of the charged particle with rigid symmetries generated by the group action $f_{g}$ of the group $G$, we now turn to examine the

\footnotetext{
${ }^{1}$ If $M$ is a two-dimensional manifold, it is always possible to arrange such that $L$ be a spin bundle and therefore the wave functions $\psi$ are (commuting) spinors
} 
quantum mechanics of the charged particle with local symmetries generated by gauging the subgroup $G^{\prime}$ of $G$. This theory described by the Hamiltonian (2.19) is quantised in a similar way as the model with rigid symmetries. However in this case the charges $Q_{a^{\prime}}$ are first class constraints and after quantisation they should annihilate the wave functions of the physical subspace $H_{\text {phys }}$ of the total Hilbert space $L^{2}(\Gamma(L), d \mu)$ of the corresponding theory with the rigid symmetries. $H_{\text {phys }}$ consists on the wave functions $\psi \in L^{2}(\Gamma(L), d \mu)$ that lie in the trivial representation of $G^{\prime} \subseteq G$ as subrepresentation in $D$ and it is the Hilbert space of the gauged charged particle. For a general line bundle $L$ and a group action $f_{g}, D$ does not necessarily contain copies of the trivial representation of $G^{\prime}$. If this is the case, $H_{\text {phys }}$ is an empty space, i.e. the theory does not have a physical Hilbert space and therefore is anomalous. An example of this phenomenon will be given in Sect. 6 .

\section{Anomalies}

In Sect. 3, it was shown that the symmetries of a charged particle generated by a group action $f_{g}$ of a group $G$ on a manifold $M$ are anomalous whenever there are obstructions to the existence of a lifting $f_{g}^{\uparrow}$ of $f_{g}$ to a principal $U(1)$ bundle $P=P(M, U(1), \pi)$ over $M$.

In the first part of this section, we study the local anomalies associated with the group action $f_{g}$ of a compact and connected Lie group $G$ on $M$ and in the second part, we examined the global anomalies which correspond to the group action $f_{g}$ of a disconnected group $G$. The latter are similar to the global anomalies described in ref. [19].

$4.1 G$ Connected. For a compact and connected group $G$, the obstructions to lifting the group action $f_{g}$ to the principal $U(1)$ bundle $P$ over $M$ are represented as follows: let $c_{1}(P)$ be the first Chern class of $P$. Then from the Kunneth formula, we get

$$
f^{*}\left(c_{1}(P)\right)=1 \otimes c_{1}(P)+a+f_{0}^{*} c_{1}(P) \otimes 1,
$$

where $f: G \times M \rightarrow M$ is the group action of $G$ on $M$ and $f_{0}: G \rightarrow M$ is given by $f_{0}(g):=f\left(g, x_{0}\right) ; x_{0}$ is a point of $M$. In general, the classes $a \in H^{1}\left(G, H^{1}(M, Z)\right)$ and $f_{0}^{*} c_{1}(P) \in H^{2}(G, Z)$ are different from zero and they serve as the obstructions to lifting the group action $f_{g}$ to the principal $U(1)$ bundle $P$. (For more details see reference [7] and Appendix A3.)

Therefore the anomalies are represented by the classes $a$ and $f_{0}^{*} c_{1}(P)$. Moreover observe that up to torsion elements in the cohomology of the group $G, a$ and $f_{0}^{*} c_{1}(P)$ can be related to the Lie algebra cohomology cocycles $\pi$ and $\tau$ given by Eqs. (2.15) and (2.16) correspondingly.

Any compact and connected Lie group $G$ can be decomposed as $G=\left(G_{a} \times G_{s}\right) / \Gamma$, where $\Gamma$ is a finite subgroup of the center of the product $G_{a} \times G_{s}$. $G_{a}$ is a product of $U(1)$ groups and $G_{s}$ is a product of simple, simply connected, and compact Lie groups. Using this decomposition of $G$, we can study the anomalies $a$ and $f_{0}^{*} c_{1}(P)$ for each component of $G$ separately. Indeed, if $G=G_{s}$ semisimple, simply connected, and compact Lie group, the group action $f_{g}$ of $G$ always lifts. This follows from the observation that the first and second cohomology of $G$ vanish with 
arbitrary coefficients, i.e. $H^{1}\left(G, H^{1}(M, Z)\right)=H^{2}(G, Z)=0$. Therefore the classes $a \in H^{1}\left(G, H^{1}(M, Z)\right)$ and $f_{0}^{*} c_{1}(P) \in H^{2}(G, Z)$ are equal to zero.

Now let $G=G_{a}=(U(1))^{n}$. In this case $H^{1}\left(G, H^{1}(M, Z)\right)$ and $H^{2}(G, Z)$ are different from zero and there are potential obstructions to lifting $U(1)$ group actions to principal $U(1)$ bundles. The obstructions $a \in H^{1}\left(G, H^{1}(M, Z)\right)$ and $f_{0}^{*} c_{1}(P) \in H^{2}(G, Z)$ are given in terms of the cocycles $\pi$ and $\tau$ (Eqs. (2.15) and (2.16)). This is because $H^{1}\left(G, H^{1}(M, Z)\right)$ and $H^{2}(G, Z)$ do not have torsion terms. An example of an obstruction to lifting a $(U(1))^{n}$ group action is the following: let $G=U(1), M=$ $S^{1} \times S^{1}$ and $f_{g}$ the diagonal action of $U(1)$ on $M$. It can be shown that $f_{g}$ does not lift to any non-trivial $U(1)$ principal bundle over $M$. This is in agreement with the results of refs. $[2,3]$.

To study the effects that the finite group $\Gamma$ has on the obstructions, we assume that the group action $h=f(j \times 1)$ of the group $G_{a} \times G_{s}$ on $M$ lifts to a principal $U(1)$ bundle $P=P(M, U(1), \pi)$, where $f$ is the action of the group $G=\left(G_{a} \times G_{s}\right) / \Gamma$ on $M$ and $j$ is the projection $j: G_{a} \times G_{s} \rightarrow G$. To find the principal $U(1)$ bundles $P=P(M, U(1), \pi)$ over $M$ that admit a lifting of the action $h$ of the group $G_{a} \times G_{s}$ but they do not admit a lifting of the group action $f$ of $G$ consider the commutative diagram

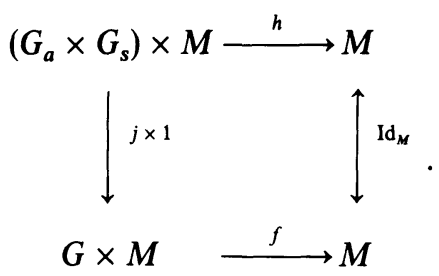

The induced commutative diagram on the second cohomology is

$$
\begin{gathered}
H^{2}\left(\left(G_{a} \times G_{s}\right) \times M, Z\right) \stackrel{h^{*}}{\longleftarrow} H^{2}(M, Z) \\
\prod_{(j \times 1)^{*}} \coprod_{\text {Id }} \\
H^{2}(G \times M, Z) \stackrel{f^{*}}{\longleftarrow} H^{2}(M, Z)
\end{gathered}
$$

Since the principal $U(1)$ bundle $P=P(M, U(1), \pi)$ admits a lifting of $h$, it implies (Appendix A3) that $h^{*} c_{1}(P)=1 \otimes c_{1}(P)$. However from the Kunneth formula, we get $f^{*} c_{1}(P)=1 \otimes c_{1}(P)+a+f_{0}^{*} c_{1}(P) \otimes 1$ (Eq. (4.1)) and from the commutativity of the diagram (4.3), $(j \times 1)^{*} a=0$ and $j^{*} f_{0}^{*} c_{1}(P)=0$. Therefore, the obstructions to lifting the group action $f$ of $G$ while the group action $h$ of $G_{a} \times G_{s}$ lifts to the principal $U(1)$ bundle $P$ with first Chern class $c_{1}(P)$ are elements $\operatorname{of} \operatorname{Ker}(j \times 1)^{*}$ restricted on $H^{1}\left(G, H^{1}(M, Z)\right)$ and $\operatorname{Ker} j^{*}$ restricted on $H^{2}(G, Z) . \operatorname{Ker}(j \times 1)^{*}$ restricted on $H^{1}\left(G, H^{1}(M, Z)\right)$ and $\operatorname{Ker} j^{*}$ restricted on $H^{2}(G, Z)$ are easily calculated in some special cases.

For example let $G$ be a simple, compact Lie group and $\Gamma$ be a subgroup of the centre of $G_{s}$ such that $G=G_{s} / \Gamma$. Since $H^{1}(G, Z)$ is equal to zero, $\operatorname{Ker}(j \times 1)^{*}$ restricted on $H^{1}\left(G, H^{1}(M, Z)\right)$ is equal to zero and the obstruction $a$ always vanishes. However $\operatorname{Ker} j^{*}$ restricted on $H^{2}(G, H)$ is equal to $\Gamma$; this can be seen 
by observing that for the simply connected, simple, and compact Lie groups the second cohomology vanishes and $H^{2}(G, Z)=\Gamma$. For example the standard action of $S O(3)$ on $S^{2}$ does not lift to the Hopf fibration over $S^{2}$ or any other principal $U(1)$ bundle over $S^{2}$ with odd first Chern number although the standard action of $S U(2)$ on $S^{2}$ lifts to all principal $U(1)$ bundles over the sphere. The obstruction class to lifting the action of $S O(3)$ group is given by the non-trivial element of the cohomology group $H^{2}(S O(3), Z)=Z_{2}$.

To give another example, we take $G=U(1)$ and $G_{a}$ the $Z_{p}$ covering of $G$. If $w$ is the generator of $H^{1}(G, Z)$ then $j^{*} w=p \tilde{w}$, where $\tilde{w}$ is the generator of $H^{1}\left(G_{a}, Z\right)$. Thus $\operatorname{Ker}(j \times 1)^{*}$ restricted on $H^{1}\left(G, H^{1}(M, Z)\right)$ is equals to zero.

$4.2 G$ Disconnected. Suppose that $G$ be a disconnected and compact group acting on a manifold $M$ with a group action $f_{g}$. The Hattori-Yoshida theorem [6] gives the necessary and sufficient conditions to lifting the group action $f_{g}$ of a compact Lie group $G$ on $M$ to any principal $U(1)$ bundle $P=P(M, U(1), \pi)$ over $M$. Indeed the group action $f_{g}$ of $G$ lifts to $P$, if and only if $f_{g}^{*} P \cong P, \forall g \in G$ and $P$ is the pullback of a principal $U(1)$ bundle $Q=Q\left(M_{G}, U(1), \pi^{\prime}\right)$ over $M_{G}:=E G \times{ }_{G} M$ with the inclusion map of $M$ in $M_{G} . E G=E G(B G, G, p)$ is the universal classifying bundle of the group $G$ with projection $p$. If $f_{g}^{*} P \cong P, \forall g \in G$, the obstructions of a principal $U(1)$ bundle $P$ over $M$ to be the pullback of a principal $U(1)$ bundle $Q$ over $M_{G}$ are elements of the cohomology groups $H^{3}(B G, Z)$ and $H^{2}\left(B G, H^{1}(M, Z)\right)$ (Appendix A2).

If the group $G$ is connected, the obstructions described by the Hattori-Yoshida theorem can be related to the cohomology classes $a$ and $f_{0}^{*}\left(c_{1}(P)\right)$ of Eq. (4.1). For the proof of this statement see ref. [7] and Appendices A2 and A3.

Let $G_{0}$ be the connected component of $G$ that contains the identity element $e$ of $G$. The sequence

$$
1 \longrightarrow G_{0} \stackrel{i}{\rightarrow} G \longrightarrow \Delta \rightarrow 1
$$

is exact where $\Delta$ is a finite but not necessarily abelian group. To study purely global anomalies, let us assume that the group action $h=(i \times 1) f$ of $G_{0}$ on $M$ lifts to the principal $U(1)$ bundle $P=P(M, U(1), \pi)$ over $M$ and $f_{g}^{*} P \cong P, \forall g \in G$. From the commutative diagram

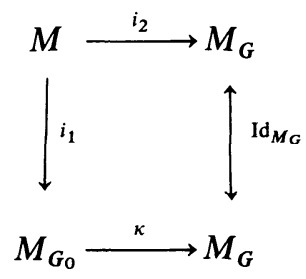

and the induced diagram on the second cohomology

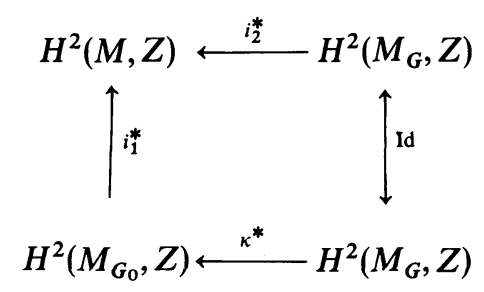


we can show that the principal $U(1)$ bundles $P$ over $M$ that accept a lifting of the action $h$ of $G_{0}$ but they do not admit a lifting of the group action $f$ of $G$ are elements of the $H^{2}\left(M_{G_{0}}, Z\right)-\operatorname{Im} k^{*} . i_{1}\left(i_{2}\right)$ is the inclusion of $M$ in $M_{G_{0}}\left(M_{G}\right)$.

There are two sources of global anomalies. The first one appears whenever the principal $U(1)$ bundle $P=P(M, U(1), \pi)$ is not isomorphic to the principal $U(1)$ bundle $f_{g}^{*} P$ for some $g \in G$. An example of this type of global anomaly was given in ref. [5]. A necessary condition for the existence of this type of global anomaly is that $H^{2}(M, Z)$ has torsion. Indeed, let $M=S^{2} \times R P(n) \times R P(n)$, where $n \geqq 3$ is an odd integer, $R P(n)$ is the real projective space and $G=S U(2) \times Z_{2}$. SU(2) acts on $S^{2}$ with the standard action and the non-trivial element $s$ of $Z_{2}$ interchange the two projective subspaces of $M$. From the Kunneth formula $H^{2}(M, Z)=Z \oplus$ $Z_{2} \oplus Z_{2}$ and it is generated by the elements $z, \alpha$ and $\beta$, where $z$ is a torsion free element, and $\alpha, \beta$ are torsion elements. The non-trivial element $s$ of $Z_{2}$ in $G$ induces the following transformation on $H^{2}(M, Z)$ :

$$
f_{(1, s)}^{*}(\alpha)=\beta, \quad f_{(1, s)}^{*}(\beta)=\alpha, \quad \text { and } \quad f_{(1, s)}^{*}(z)=z .
$$

The connected component $G_{0}\left(G_{0}=S U(2)\right)$ of $G$ lifts to all principal $U(1)$ bundles over $M$. Both cohomology groups $H^{3}(B G, Z)$ and $H^{2}\left(B G, H^{1}(M, Z)\right)$ vanish.

Let $F$ be the curvature 2-form of a $U(1)$-principal bundle $P=P(M, U(1), \pi)$ invariant under the action of the group $G$ (Eq. (2.7)), and $[F]$ be the invariant, torsion free representative of $F$ in $H^{2}(M, Z)$. The curvature $F$ does not completely classify the principal bundle $P$ since both principal bundles with first Chern classes $[F]$ and $[F]+\alpha$ may have the same curvature form $(\alpha$ and $\beta$ correspond to flat principal bundles). Thus, if $c_{1}(P)=[F]+\alpha, f_{(1, s)}^{*} c_{1}(P)=[F]+\beta \neq[F]+\alpha$, and the action $f_{g}$ does not lift to $P$ even though its curvature two-form $F$ is invariant. Finally, observe that the group $G$ lifts to the principal $U(1)$ bundles over $M$ with first Chern classes, $n z$ and $n z+\alpha+\beta, n$ integer.

The second type of global anomaly is due to the existence of obstructions to lifting the group action $f_{g}$ of $G$ to the principal $U(1)$ bundle $P=P(M, U(1), \pi)$ which are elements in $H^{3}(B G, Z)$ and $H^{2}\left(B G, H^{1}(M, Z)\right)$ even though the connected part $G_{0}$ of $G$ lifts (the local anomalies vanish) and $f_{g}^{*} P \cong P, \forall g \in G$. To give an example of this type of global anomaly, we set $G=Z_{p}$. Then we take $M=S^{1} \times S^{1}$ and the group action $f_{k}(\phi, \theta)=(\phi+(2 / p) \pi, \theta+(2 / p) \pi) \bmod 2 \pi$, where $k$ is the generator of $Z_{p}$ and $0<\phi, \theta \leqq 2 \pi$ are the standard coordinates on $M$. $Z$ acts freely on $M$ and from the fibration

$$
E G \rightarrow M_{G} \rightarrow M / G
$$

we deduce that $M_{G}$ is homotopic to $M / G . M / G$ is diffeomorphic to $S^{1} \times S^{1}$. The inclusion $i: M \rightarrow M_{G}$ is homotopic to the standard projection $M \rightarrow M / G$ and the induced map on the cohomology is $i^{*} w=p \tilde{w}$ and $i^{*} z=p \tilde{z}$, where $\{w, z\}$ are the generators of $H^{1}(M / G, Z)$ and $\{\tilde{w}, \tilde{z}\}$ are the generators of $H^{1}(M, Z)$. The second cohomology $H^{2}(M, Z)\left(H^{2}(M / G, Z)\right)$ group is generated by $\tilde{w} \cup \tilde{z}(w \cup z)$, where $\cup$ is the cup product. This implies that $f_{g}^{*} P \cong P, \forall g \in G$ and $i^{*}(w \cup z)=p^{2}(\tilde{w} \cup \tilde{z})$. Therefore $\operatorname{Im} i^{*}$ is generated by $p^{2}(\tilde{w} \cup \tilde{z})$. Thus all the principal $U(1)$ bundles over the torus $M$ with first Chern number $n$ such that $n \bmod p^{2} \neq 0$ do not admit a lifting of the group action $f$ of $Z_{p}$. 


\section{Supersymmetric Charged Particles}

Let $\xi$ be a vector bundle with connection $\Omega$ over a Riemannian manifold $M$ with metric g. The covariant derivative $\nabla$ of $\xi$ is compatible with a fibre metric $h$. The Lagrangian of $N=1$ supersymmetric sigma model locally on $M$ is given [18] by

$$
L_{\alpha}=(1 / 2) \mathbf{g}_{i j \alpha} D \phi^{i} \partial_{t} \phi^{j}+(i / 2) h_{A B \alpha} \lambda^{A} \hat{\nabla} \lambda^{B}+b_{i \alpha} D \phi^{i},
$$

where $\phi$ is a real $C^{\infty}$ function (superfield) from the flat superspace $\Xi^{1 / 1}$ to the manifold $M, \lambda$ is a $C^{\infty}$ section of the vector bundle $\phi^{*} \xi$ over $\Xi^{1 / 1}, D^{2}=i \partial_{t}$, $A, B=1, \ldots, \operatorname{rank} \xi$ and $\hat{\nabla}=D \phi^{i} \nabla_{i}$. The equations of motion of the Lagrangian (5.1) are invariant under a group action $f_{g}$ of a group $G$ on manifold $M$ provided that Eqs. (2.6) and (2.7) hold, and the connection $\Omega$ is invariant up to a gauge transformation [23]. To quantise this theory, we proceed along the same lines as in the case of the charged particle. The wave functions of this theory are the square integrable sections of the vector bundle $\eta=S \otimes L \otimes \varepsilon$ over $M$, where $S$ is a spin bundle over $M, L$ is a complex line bundle over $M$ with connection $b$ and $\varepsilon$ is either the spin bundle of the vector bundle $\xi$, if $\varepsilon$ is a real vector bundle, or $\varepsilon=\xi^{2}$, if $\xi$ is a hermitian vector bundle. An inner product in $\Gamma(\eta)$ is defined as in Eq. (3.5). The theory is anomalous, if the manifold $M$ does not admit a spin structure $\left(w_{2} \neq 0\right)[18]$ (we assume that if $\xi$ is real, it admits a spin structure $\left.w_{2}(\xi)=0\right)$. This anomaly may be cancelled by taking the wave functions to be sections of $\eta=S_{c} \otimes \varepsilon$ vector bundle, where $S_{c}$ is a $\operatorname{Spin}_{c}(n)=\operatorname{Spin}(n) \times_{z_{2}} U(1)$ vector bundle [13]. However, another anomaly may appear which is the obstruction to the existence of a $\mathrm{Spin}_{c}$ structure $[20]\left(w_{3} \neq 0\right)$. A further modification is possible, the wave functions of the theory may be taken to be sections of a vector bundle $\eta$ with structure group $\operatorname{Spin}_{G}(n)=\operatorname{Spin}(n) \times_{Z_{2}} G[16]$. Even after this modification, the theory may remain anomalous. The anomalies are due to obstructions to the existence of $\operatorname{Spin}_{G}$ structures [21]. The Hamiltonian operator of a supersymmetric charged particle after a choice of an ordering ambiguity is the Laplace-Beltrami operator of $M$ twisted with a connection of the vector bundle $\eta$.

As in the case of the charged particle, the necessary and sufficient condition to implement the symmetries generated by the group action $f_{g}$ with unitary transformations on the Hilbert space of the quantum theory is the existence of a lifting $f_{g}^{\uparrow}$ of $f_{g}$ on $\eta$. This implies that the group action $f_{g}$ must lift to the associated principal bundle $P(M, K, \pi)$ of $\eta$. However in this case, the structure group of $P(M, K, \pi)$ is not necessarily isomorphic to $U(1)$ (or a product of $U(1)$ ) and the Hattori-Yoshida theorem does not apply.

Next suppose that $P(M, K, \pi)$ admits a lifting $f_{g}^{\uparrow}$ of a group action $f_{g}$. If this is the case, $P(M, K, \pi)$ is the pullback of a principal bundle $P_{0}\left(M_{G}, K, \pi^{\prime}\right)$ over $M_{G}=E G \times{ }_{G} M$ with the inclusion map of $M$ in $M_{G}$ (Appendix A1). Thus the necessary conditions to the existence of $f_{g}^{\uparrow}$ can be derived by studying the obstructions to extend the classifying map $t: M \rightarrow B K$ of the principal bundle

\footnotetext{
${ }^{2}$ The wave functions of the supersymmetric charged particle within hermitian vector bundle $\xi$ are sections of the vector bundle $S \otimes L \otimes \Lambda \xi$, where $\Lambda \xi=\Sigma_{n=0} \Lambda^{n} \xi$. However the projection from $S \otimes L \otimes \Lambda \xi$ onto $S \otimes L \otimes \xi$ can be performed without violating the supersymmetry of the theory [18]
} 
$P(M, K, \pi)$ to $M_{G}$. The extension problem can be described by the following commutative diagram

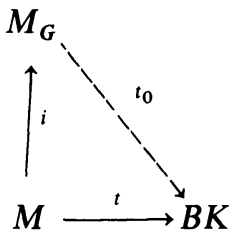

The map $t_{0}$ must be specified up to a homotopy equivalence and it is the classifying map of a principal bundle $P_{0}\left(M_{G}, K, \pi^{\prime}\right)$ over $M_{G}$. Given the classifying map $t$ of $P(M, K, \pi)$ and the inclusion $i$ of $M$ in $M_{G}$, to construct $t_{0}$ is a standard extension problem and it can be solved by using topological perturbation theory. The obstructions to extend the map $t$ to $t_{0}$ correspond to anomalies in the quantum mechanics of the supersymmetric charged particle:

It is possible to describe all the obstructions to lifting group actions to principal fibre bundles $P(M, K, \pi)$ with non-abelian structure group provided that $P(M, K, \pi)$ has some additional structure. For example, the base space $M$ is a homogeneous space or $P(M, K, \pi)$ admits a flat connection. The former case is studied in Sect. 6 .

The principal bundles $P(M, K, \pi)$ which admit flat connections are of general interest. Indeed flat bundles are useful in the study of Aharonov-Bohm effect [11] $(K=U(1))$ and the understanding of (non-abelian) $\theta$-structures in quantum mechanics. The principal flat bundles $P=P(M, K, \pi)(K$ non-abelian) over $M$ correspond to the elements of $\operatorname{Hom}\left(\pi_{1}\left(M, x_{0}\right), K\right)$ up to conjugations where $x_{0}$ is a point in $M(K=U(1)$ for principal flat $U(1)$ bundles). Let $\alpha$ be an element of $\operatorname{Hom}\left(\pi_{1}\left(M, x_{0}\right), K\right)$ then the principal bundle $P(M, K, \pi)$ corresponding to the group homomorphism $\alpha$ is an associated bundle of the principal bundle $P\left(M, \pi_{1}\left(M, x_{0}\right)\right.$ / $\left.\operatorname{Ker} \alpha, \pi^{\prime}\right)$ with discrete fibers. Indeed $P(M, K, \pi)=P\left(M, \pi_{1}\left(M, x_{0}\right) / \operatorname{Ker} \alpha, \pi^{\prime}\right) \times{ }_{\alpha} K$. To study the lifting of the group action $f_{g}$ on the flat principal bundle $P(M, K, \pi)$, it is enough to examine the liftings of $f_{g}$ on $P\left(M, \pi_{1}\left(M, x_{0}\right) / \operatorname{Ker} \alpha, \pi^{\prime}\right)$. The actions of simply connected groups always lift to all principal bundles with discrete fibers. More details are given in Appendix A4.

\section{The Charged Particle on $G / H$ Spaces}

In the first part of this section, we study the Hilbert space $\left(L^{2}(\Gamma(\varepsilon), d \mu)\right) L^{2}(\Gamma(L), d \mu)$ of (supersymmetric) charged particles propagating on a homogeneous space $G / H$ which are invariant under the left action of $G$ on $G / H$. $G$ is restricted to be compact and connected. Then we gauge a subgroup $G^{\prime}$ of the group $G$. Using the representation theory of $G$ on the Hilbert space $L^{2}(\Gamma(L), d \mu)$, we give an example of a gauged charged particle with empty physical Hilbert space, i.e. an example of a gauged charged particle anomaly.

The principal bundles $P=P(M, K, \pi)$ that admit a lifting $l_{g}^{\uparrow}$ of the left action $l_{g}$ of $G$ on $M=G / M$ correspond to group homomorphisms $\alpha: H \rightarrow K ; P=G \times{ }_{\alpha} K$ (Appendix A4). Let $\varepsilon_{0}$ be a finite dimensional vector space that carries a unitary representation $u$ of the group $K$. Then using the lifting $l_{g}^{\uparrow}$ of $G$ on $P(M, K, \pi)$, we can construct a unitary representation of $G$ on the square integrable sections 
$L^{2}(\Gamma(\varepsilon), d \mu)$ of the vector bundle $\varepsilon=P x_{u} \varepsilon_{0}$, where $d \mu$ is the invariant measure in $G / H$. This is possible because given $l_{g}^{\dagger}$ there is a lifting $l_{g, u}^{\uparrow}$ of $G$ on $\varepsilon$ defined by

$$
l_{g, u}^{\uparrow}[p, v]=\left[l_{g}^{\uparrow}(p), v\right], \quad p \in P \text { and } v \in \varepsilon_{0} .
$$

Since $G$ is compact, the representation of $G$ on $L^{2}(\Gamma(\varepsilon), d \mu)$ decomposes in finite dimensional unitary irreducible representations (for a review see reference [24]). Indeed observe that the sections $L^{2}(\Gamma(\varepsilon), d \mu)$ can be identified with the square integrable functions $L^{2}\left(C\left(G, \varepsilon_{0}, u, \alpha\right), d \mu\right)$ from $G$ to $\varepsilon_{0}$ that satisfy the property,

$$
\psi(g h)=u\left(\alpha\left(h^{-1}\right)\right) \psi(g) \quad \forall h \in H .
$$

We have identified $\left.\varepsilon\right|_{e H}$ with $\varepsilon_{0}$. Given an irreducible representation $D_{\gamma}$ of the group $G$ on the vector space $V_{\gamma}$, we can construct a function $\psi_{\gamma}$ on $G$ that satisfies (6.2). Indeed let $F_{\gamma}: V_{\gamma} \otimes \operatorname{Hom}_{H}\left(V_{\gamma}, \varepsilon_{0}\right) \rightarrow C\left(G, \varepsilon_{0}, u, \alpha\right)$ such that $F_{\gamma}(v \otimes B)(g)=B\left(D_{\gamma}\left(g^{-1}\right) v\right)$, where $B \in \operatorname{Hom}_{H}\left(V_{\gamma}, \varepsilon_{0}\right), v \in V_{\gamma}$ and $g \in G$. Then $\psi_{\gamma}:=F_{\gamma}(v \otimes B)$. $\operatorname{Hom}_{H}\left(V_{\gamma}, \varepsilon_{0}\right)$ is the space of linear functions $B$ from $V_{\gamma}$ to $\varepsilon_{0}$ that have the property $B\left(D_{\gamma}(h) v\right)=$ $u(\alpha(h)) B(v)$. Finally, it can be shown that

$$
\Sigma_{\gamma} F_{\gamma}\left(V_{\gamma} \otimes \operatorname{Hom}_{H}\left(V_{\gamma}, \varepsilon_{0}\right)\right)
$$

spans $L^{2}\left(C\left(G, \varepsilon_{0}, u, \alpha\right), d \mu\right)$, where the sum is over the set of irreducible unitary representations $D_{\gamma}$ of the group $G$. Suppose that the unitary representation $u_{\alpha}:=u \alpha$ of the group $H$ on $\varepsilon_{0}$ is irreducible. From Frobenious reciprocity theorem, the multiplicity $d(\gamma)$ of the irreducible representation $D_{\gamma}$ of $G$ in the representation $D$ (Eq. (3.6)) (acting on the Hilbert space $L^{2}\left(C\left(G, \varepsilon_{0}, u, \alpha\right), d \mu\right)$ ) is equal to the multiplicity of the representation $u_{\alpha}$ of the group $H$ as subrepresentation of $\left.D_{\gamma}\right|_{H} .\left.D_{\gamma}\right|_{H}$ is the restriction of $D_{y}$ on the group $H$. For example if $H=\{e\}$ and $\varepsilon_{0}=\mathbf{C}$, the action of $G$ on $L^{2}(\Gamma(\varepsilon), d \mu)$ gives the regular representation of $G$ that contains all the unitary irreducible representations $D_{\gamma}$ of $G$ with multiplicity $d(\gamma)$ equals to the dimension of $D_{\gamma}$.

To find the eigenvalues of the Hamiltonian operator $\hat{H}(3.1)$ on the Hilbert space $L^{2}(\Gamma(L), d \mu)$ invariant under the left action of $G$ on $G / H$, we assume that $G$ is semisimple, compact, and connected Lie group, and the metric on $G / H$ is the metric induced by the Killing-Cartan metric of Lie $G$. Then, the eigenvalues (up to a constant) of the Hamiltonian operator $\hat{H}(3.1)$ are proportional to quadratic Casimir of the group $G$ of every irreducible representation $D_{\gamma}$ that appears in the decomposition (6.3). Since this theorem to true for all the twisted Laplace-Beltrami operators [24], we can also use it to calculate the eigenvalues of the Hamiltonian operator of supersymmetric charged particle. The quadratic Casimir $c(\gamma)$ of the irreducible representation $D_{\gamma}$ with highest weight $\Lambda_{\gamma}$ is given by

$$
c(\gamma)=\left\langle\Lambda_{\gamma}+\rho, \Lambda_{\gamma}+\rho\right\rangle-\langle\rho, \rho\rangle,
$$

where $\langle$,$\rangle is the Cartan metric on Lie G$ and $\rho$ is half the sum of positive roots of Lie $G$.

The physical Hilbert space $H_{\text {phys }}$ of a gauged charged particle is the subspace of $L^{2}(\Gamma(L), d \mu)$ that carries the (copies) trivial representation of $G^{\prime} \cong G$ as subrepresentation of $D(3.6)$, where $G^{\prime}$ is a subgroup of $G$ which has been gauged. If the line bundle $L$ is not topologically trivial, the trivial representation of $G^{\prime}$ may not be a subrepresentation of $D$. To give an example of a gauged charged particle model with physical Hilbert space $H_{\text {phys }}$ empty, we set $M=S^{2}=S U(2) / U(1)$, 
$G^{\prime}=S U(2)$ and $L$ the spin bundle $L_{s}$ over $S^{2} . L_{s}$ is an associated complex line bundle of the principal Hopf fibration with the faithful representation of $U(1)$ on $\mathbf{C}$. The left action of $S U(2)$ on $S^{2}$ lifts to all $U(1)$ principal bundles over $S^{2}$ and the Hopf fibration corresponds to the identity group homomorphism $\alpha$ from $H=U(1)$ to $K=U(1)$. Finally using the decomposition (6.3) for the sections $\Gamma\left(L_{s}\right)$ of the spin line bundle $L_{s}$, we can prove that the trivial representation of $S U(2)$ is not a subrepresentation of $D(3.6)$ on $L^{2}\left(\Gamma\left(L_{s}\right), d \mu\right)$; $d \mu$ is the invariant measure on $S^{2}$. This implies that the physical Hilbert space $H_{\text {phys }}$ of the theory is empty.

\section{Conclusions}

We can arrange such that some of the anomalies studied in Sect. 4 are cancelled. This can be achieved by altering appropriately the group $G$, the group action $f_{g}$ and (or) the principal $U(1)$ bundle $P$. For example, let $G$ be a connected, semisimple Lie group and assume that the classical symmetries of the charged particle generated by the group action $f_{g}$ on the manifold $M$ are anomalous. These anomalies are cancelled, if we consider the lifting of the induced action of the universal cover of $G$ on $M$. Alternatively, it might be possible to choose a $U(1)$ principal bundle such that the symmetries generated by the group action $f_{g}$ of $G$ are not anomalous. However, this might require an additional quantisation of the coupling constant of the interaction term, i.e. a refinement of Dirac's quantisation condition. An example of this phenomenon was given in Sect. 4. If $G$ is disconnected, a similar cancellation mechanism for the global anomalies may be devised.

Another interesting phenomenon is the $\theta$-structure effects due to a minimal substitution of a (flat) connection in the momentum operator of a theory (refs. [11] and [12]). Having fixed the connection $b$ for the charged particle, we studied a given sector of this theory. To examine other sectors, we substitute the connection $b$ in the hamiltonian operator $\hat{H}$ (3.1) with another connection $A$. This change may alter even the domain that the operator $\hat{H}$ acts. To test whether the symmetries of the charged particle generated by the action $f_{g}$ of the group $G$ are implemented in the sector represented with the connection $A$, we should repeat the analysis of Sects. 3 and 4 for the Hamiltonian operator $\hat{H}$ with connection $A$. It is possible that the symmetries generated by the group $G$ can be implemented by unitary transformations in the Hilbert space of one sector but not in the Hilbert space of another.

In conclusion, the quantum mechanics of the charged particle propagating on a manifold $M$ with a group action $f_{g}$ of the group $G$ was studied. It was shown that these symmetries may be anomalous. There are anomalies both of global and local type. For the particle with rigid symmetries these anomalies manifest themselves as obstructions to lifting group actions to $U(1)$ principal bundles. Several examples of obstructions were given and the nature of these obstructions was illustrated.

In the case of gauged charged particle, apart from the anomalies that appear in the charged particle with rigid symmetries, there is an additional anomaly whenever the physical Hilbert space is empty. An example of this anomaly was given in Sect. 7 associated with the gauging of the left action of $S U(2)$ on $S^{2}$ for a charged particle coupled to a $U(1)$ connection of the Hopf fibration over $S^{2}$. 
The supersymmetric charged particles were also considered. It was shown that the classical symmetries of this theory generated by a group action $f_{g}$ may be anomalous. These anomalies were related to obstructions to lifting the group action $f_{g}$ on a principal bundle $P$ over $M$ with fiber a non-abelian group $K$. It was observed that some of these obstructions can be identified with the obstructions of a topological extension problem.

\section{Appendices}

A1. Liftings and Obstructions. Let $P=P(X, K, \pi)$ be a principal bundle with fibre a compact and connected Lie group $K$ over a compact, path connected, orientable manifold $X$ without boundary. $\pi: P \rightarrow X$ is the projection of $P$ onto $X$. Moreover, let $f_{g}$ be a group action of the compact Lie group $G$ on $X$. The lifting $f_{g}^{\uparrow}$ of the group action $f_{g}$ is a group action of $G$ on $P$ such that $\pi f_{g}^{\uparrow}=f_{g} \pi, \forall g \in G$.

The group action $f_{g}$ defines a continuous map $\rho: G \rightarrow \operatorname{Homeo}(X)$, such that $\rho(g)=f_{g}$. Homeo $(X)$ is the set of homeomorphisms of $X$ with the compact open topology and $\rho(G):=\operatorname{Im} \rho$ is a topological group. From the definition of the lifting $f_{g}^{\uparrow}$, it implies that there is a continuous map $\rho^{\uparrow}: G \rightarrow \operatorname{Isom}(P)$ such that $\rho^{\uparrow}(g)=f_{g}^{\uparrow}$, where $\operatorname{Isom}(P)$ is the space of bundle morphisms of $P$. Denote with $\sigma$ the natural $\operatorname{map} \sigma: \operatorname{Isom}(P) \rightarrow \operatorname{Homeo}(X), \sigma$ is not necessarily surjective. Now suppose that $f_{g}^{*}(P) \cong P, \forall g \in G$, then the following sequence is exact:

$$
1 \rightarrow \operatorname{Aut}(P) \rightarrow \operatorname{Isom}_{G} P \rightarrow \rho(G) \rightarrow 1,
$$

where $\operatorname{Isom}_{G}(P):=\sigma^{-1} \rho(G)$ and $\operatorname{Aut}(P)$ is the space of bundle morphisms of $P$ that induce the identity transformation on $X$. To find a lifting $f_{g}^{\uparrow}$ of the group action $f_{g}$, it is equivalent to construct a section in (A1.1) which is a group homomorphism. If there is a section in (A1.1), then to find $f_{g}^{\dagger}$ is a standard group extension problem. A section $v$ of (A1.1) which is not necessarily a group homomorphism is called pseudolifting.

To continue, suppose that $P$ accepts a lifting $f_{g}^{\dagger}$ of the group action $f_{g}$ on $X$. Then $P$ is the pullback of a principal bundle $P_{0}\left(X_{G}, K, \pi_{0}\right)$ over $X_{G}:=E G \times{ }_{G} X$ with respect to the inclusion $i: X \rightarrow G$, where $E G(B G, G, p)$ is the universal classifying bundle of the group $G$. The bundle space $P_{0}$ is isomorphic to $E G \times{ }_{G} P$ and the projection $\pi_{0}$ is induced from the projection $\pi$ of $P$. The converse of the above statement is not necessarily true for generic principal bundles $P[7]$. However it was shown in ref. [6] that is always true in the case of principal $U(1)$ bundles.

A2. Lifting Group Actions to Principal $U(1)$ Bundles. To prove that a principal $U(1)$ bundle $P=P(M, U(1), \pi)$ over $X$ admits a lifting $f_{g}^{\dagger}$ of a group action $f_{g}$ of the compact group $G$ on $X$ if and only if $f_{g}^{*}(P) \cong P, \forall g \in G$ and $P$ is the pullback of a principal bundle over $X_{G}$ with the inclusion $i$ of $X$ in $X_{G}$, we proceed as follows: First we show that if the principal bundle $P$ is the pullback of a principal bundle over $X_{G}$ with the map $i: X \rightarrow X_{G}$, then $P$ admits a pseudolifting of the group action $f_{g}$. This implies that there is $\hat{f}_{g}: P \rightarrow P, \forall g \in G$, such that $\hat{f}_{g} \hat{f}_{g^{\prime}}=\hat{f}_{g g^{\prime}} o\left(g, g^{\prime}\right)$. $o\left(g, g^{\prime}\right)$ is the obstruction of the pseudolifting $\hat{f}_{g}$ to be a group homomorphism. It can be shown that $o$ is independent from the choice of pseudolifting and is an element of the group cohomology $H^{2}(G, \operatorname{Aut}(P)$ ) (for $U(1)$ principal bundles 
$\operatorname{Aut}(P)=C(X, U(1))$. Then it is shown that for $U(1)$ principal bundles $P(X, U(1), \pi)$ and compact groups $G$ the obstruction $o$ vanishes.

The principal $U(1)$ bundles $P=P(M, U(1), \pi)$ over any manifold $X$ are classified by the elements of $H^{2}(X, Z)$, i.e. by their first Chern class $c_{1}(P)$. Therefore, the principal $U(1)$ bundles $P$ that accept a lifting of the group action $f_{g}$ of $G$ on $X$ lie in the image $\operatorname{Im} i^{*}$ of $i^{*}: H^{2}\left(X_{G}, Z\right) \rightarrow H^{2}(X, Z)$. To describe $\operatorname{Im} i^{*}$, we consider the fibration

$$
X \stackrel{\bullet}{\longrightarrow} X_{G} \longrightarrow B G
$$

where $B G$ is the base space of the universal classifying bundle $E G(B G, G, p)$ of the group $G . \operatorname{Im} i^{*}$ consists on the elements of $H^{2}(X, Z)$ which "survive" to $E_{\infty}$, where $\left(E_{r}, d_{r}\right), r=1, \ldots, \infty$, is the Leray-Serre spectral sequence of the fibration (A2.1). Since $\operatorname{Im} i^{*} \subseteq H^{2}(X, Z)$, it is enough to find the elements of $H^{2}(X, Z)$ that they "survive" up to $E_{4}$. Thus if $c_{1}(P)$ is the first Chern class of the principal bundle $P$, then $c_{1}(P)$ is in $\operatorname{Im} i^{*}$, provided that $d_{2} c_{1}(P)=0$ and $d_{3} c_{1}(P)=0$. Since $d_{2}: H^{2}(X, Z) \rightarrow H^{2}\left(B G, H^{1}(X, Z)\right)$ and $\operatorname{Im} d_{3} \subseteq H^{3}(B G, Z)$, if there are obstructions they are elements of the cohomology groups $H^{2}\left(B G, H^{1}(X, Z)\right)$ and $H^{3}(B G, Z)$.

Given a lifting $f_{g}^{\uparrow}$ of the group action $f_{g}$ on the principal bundle $P$, we can construct another lifting $f_{g, v}^{\uparrow}$ by conjugating with any element $v$ of Aut $(P)$. Indeed

$$
f_{g, v}^{\uparrow}=v^{-1} f_{g}^{\uparrow} v
$$

For $U(1)$ principal bundles the inequivalent liftings up to Aut $(P)$ transformations are classified by the elements of $H^{1}(G, C(X, U(1)))$.

A3. Lifting Group Actions of Compact and Connected Lie Groups. Now suppose that $G$ is compact and connected and acts on the manifold $X$ with the group action $f_{g}$. It can be shown that the principal $U(1)$ bundle $P=P(M, U(1), \pi)$ is in the $\operatorname{Im} i^{*}$ if and only if $f^{*} c_{1}(P)=1 \otimes c_{1}(P)$, where $c_{1}(P)$ is the first Chern class of $P$ [7]. The map $i$ is the inclusion of the fibre in the fibration (A2.1). This statement provides a simple discription of the obstructions to lifting compact and connected Lie groups. Indeed, it is expected from the Kunneth formula that

$$
f^{*} c_{1}(P)=1 \otimes c_{1}(P)+a+f_{0}^{*} c_{1}(P) \otimes 1,
$$

where $a \in H^{1}\left(G, H^{1}(X, Z)\right)$ and $f_{0}^{*} c_{1}(P) \in H^{2}(G, Z) . \quad f_{0}: G \rightarrow X$ is defined by $f_{0}(g):=f\left(g, x_{0}\right)$, where $x_{0}$ is a given point of the space $X$. Therefore, the obstructions to lifting the group action $f_{g}$ of the compact and connected group $G$ are represented by the elements $a$ and $f_{0}^{*} c_{1}(P)$.

To prove this statement, we consider the commutative diagram

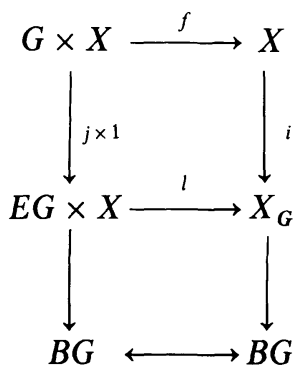


where $j$ is the inclusion of $G$ in $E G$ and $l$ is the standard projection from $E G \times X$ to $X_{G}=E G \times{ }_{G} X$. From the commutativity of (A3.2), i $f=l(j \times 1)$ and the induced map on cohomology is $f^{*} i^{*}=(j \times 1)^{*} l^{*}$. Thus if $c_{1}(P)$ is in $\operatorname{Im} i^{*}$, then $f^{*} c_{1}(P)=(j \times 1)^{*} l^{*} c_{1}(P)$. But $E G$ is contractible, therefore $f^{*} c_{1}(P)=1 \otimes c_{1}(P)$ and this proves the statement in one direction. Conversely, let us assume that $f^{*} c_{1}(P)=1 \otimes c_{1}(P)$, then we have to show that $d_{2} c_{1}(P)=d_{3} c_{1}(P)=0$, where $\left(E_{r}, d_{r}\right)$ is the Leray-Serre spectral sequence of the fibration (A2.1). Indeed, let $\left(E_{r}^{\prime}, d_{r}^{\prime}\right)$ be the Leray-Serre spectral sequence of the fibration $G \times X \rightarrow E G \times X \rightarrow B G$. The classes $1 \otimes c_{1}(P)$ survive to $E_{\infty}^{\prime}$ thus $d_{r}^{\prime}\left(1 \times c_{1}(P)\right)=0, \forall r$ integer. The differentials $d_{r}$ and $d_{r}^{\prime}$ commute with the induced maps $l_{r}$, therefore for $r=2 d_{2}^{\prime} l_{2}=l_{2} d_{2}$, where $l_{2}=1 \otimes f^{*}$ and $l_{2}$ is injective. Thus if $f^{*} c_{1}(P)=1 \otimes c_{1}(P)$, it implies that $d_{2} c_{1}(P)=0$. Similarly, it can be shown that $d_{3} c_{1}(P)=0$. This proves the statement.

A.4. Lifting the Left Action of $G$ on $G / H$. To study the necessary and sufficient conditions to lifting the left group action of $G$ on the homogeneous space $X=G / H$, where $G$ is compact and connected Lie group and $H$ is a subgroup of $G$, we let $P=P(X, K, \pi)$ be a principal $K$ bundle over $X$ which accepts a lifting $l_{g}^{\uparrow}$ of the left action $l_{g}$ of $G$ on $X$. Then we can define a group homomorphism $\alpha: H \rightarrow K$ as follows: let $e H \in G / H$, then $l_{h} e H=e H, \forall h \in H$, thus $l_{h}^{\dagger} \pi^{-1}(e H) \cong \pi^{-1}(e H)$. Given a point $p_{0} \in \pi^{-1}(e H), \forall h \in H$ there is $k \in K$ such that $l_{h}^{h}\left(p_{0}\right)=p_{0} k$. Define $\alpha(h):=k$. We observe that given the group homomorphism $\alpha, P=G \times{ }_{\alpha} K$ [9]. The converse is also true, if $P=G \times{ }_{\alpha} K$ for some group homomorphism $\alpha: H \rightarrow K$, then $P$ accepts a lifting of the left action of the group $G$ on $G / H$ which is defined by $l_{g^{\prime}}^{\dagger}[g, k]_{\alpha}=\left[g^{\prime} g, k\right]_{\alpha}$.

Finally we examine the lifting of a group action $f_{g}$ of a compact and connected group $G$ to a principal bundle with discrete fibres. The effective action $f_{g}$ of $G$ on the base space $X$ of the principal $P=P(X, Z, \pi), Z$ discrete, lifts to $P$, if and only if $f_{0 *} \pi_{1}\left(G, x_{0}\right) \subseteq \pi_{*}\left(\pi_{1}\left(P, p_{0}\right)\right)$ where $x_{0} \in X, p_{0} \in X$, and $\pi\left(p_{0}\right)=x_{0}$, and $f_{0}: G \rightarrow X$ such that $f_{0}(g):=f_{g}\left(x_{0}\right)$. If $G$ does not lift, then an extension $G^{\prime}$ of $G$

$$
1 \rightarrow \Gamma \rightarrow G^{\prime} \rightarrow G \rightarrow 1
$$

always lifts, where $\Gamma \cong \pi_{1}\left(P, p_{0}\right)[17]$. A similar statement is true if $G$ is disconnected.

Acknowledgements. I would like to thank P. S. Howe and L. Hodgkin for useful discussions. I am indebted to C. M. Hull and B. Spence for letting me know their results in ref. [14] prior to publication. This work was supported by SERC.

\section{References}

1. Dirac, P. A. M.: Proc. R. Soc. A133, 60 (1931)

2. Manton, N. S.: Ann. Phys. 159, 220 (1985)

3. Ward, R. S.: Phys. Rev. D36, 640 (1987)

4. Jackiw, R., Manton, N.: Ann. Phys. 127, 257 (1980); Jackiw, R.: In: Current Algebras and Anomalies. Singapore: World Scientific 1985

5. Papadopoulos, G.: Phys. Lett. 248B, 113 (1990)

6. Hattori, A., Yoshida, T.: Jpn J. Math. 2, 13 (1976)

7. Gottlieb, D. H.: Lifting Actions on Fibrations. Lect. Notes in Math. Vol. 757, Berlin, Heidelberg, New York: Springer 1978

8. Mostow, G. M.: Ann. Math. 73, 20 (1961) 
9. Kobayashi, S. Nomizu, K.: Foundations of Differential Geometry. Vol. II, London: Interscience 1983

10. Papadopoulos, G.: Class. Quantum Grav. 7, 41 (1990)

11. Aharonov, Y., Bohm, D.: Phys. Rev. 115, 485 (1959)

12. Isham, C. J.: Topological and Global Aspects of Quantum Theories. Relativity, Groups and Topology II, (eds.). DeWitt, B. S., Stora, R. Amsterdam: North-Holland 1984

13. Howe, P. S., Townsend, P. K.: Chern-Simons Quantum Mechanics. CERN/TH-5307/89

14. Hull, C. M., Spence, B.: Nucl. Phys. B353, 379 (1991)

15. Harnad, J., Shinder, S., Vinet, L.: J. Math. Phys. 21, 2719 (1980)

16. Howe, P. S.: Elliptic operators and supersymmetry. Supermembranes and Physics in $2+1$ Dimensions. (eds.). Duff, M. J., Pope, C. N., Sezgin, E. (eds). Singapore: World Scientific 1990

17. Bredon, G. E.: Introduction to compact transformation groups. New York and London: Academic Press 1972

18. Friedan, D., Windey, P.: Nucl. Phys. B235 [FS11], 395 (1984)

19. Witten, E.: Phys. Lett. 117B, 324 (1982)

20. Killingback, T. P., Rees, E. G.: Class. Quantum Grav. 2, 433 (1985)

21. Avis, S. J., Isham, C. J.: Commun. Math. Phys. 72, 103 (1980)

22. Hochschild, G., Serre, J.-P.: Ann. Math. 57, 591 (1953)

23. Papadopoulos, G.: Phys. Lett. 238, 75 (1990)

24. Mackey, G.: Unitary Group Representation in Physics, Probability, and Number Theory. Reading, MA: Addison-Wesley 1989; Wallach, N. R.: Harmonic Analysis on Homogeneous Spaces. New York: Marcel Dekker 1973

Communicated by N. Yu. Reshetikhin 